\title{
Optical cavity mode dynamics and coherent phonon generation in high- $Q$ micropillar resonators
}

\author{
S. Anguiano, ${ }^{1}$ A. E. Bruchhausen, ${ }^{1}$ I. Favero, ${ }^{2}$ I. Sagnes,${ }^{3}$ A. Lemaître, ${ }^{3}$ N. D. Lanzillotti-Kimura, ${ }^{3}$ and A. Fainstein ${ }^{1, *}$ \\ ${ }^{1}$ Centro Atómico Bariloche \& Instituto Balseiro, CNEA, CONICET, 8400 San Carlos de Bariloche, Río Negro, Argentina \\ ${ }^{2}$ Matériaux et Phénomènes Quantiques, Université Paris Diderot, CNRS-UMR 7162, Sorbonne Paris Cité, 10 rue Alice Domon et Léonie \\ Duquet, 75013 Paris, France \\ ${ }^{3}$ Centre de Nanosciences et de Nanotechnologies, CNRS, Université Paris-Sud, Université Paris-Saclay, C2N Marcoussis, \\ 91460 Marcoussis, France
}

(Received 9 May 2018; published 9 July 2018)

\begin{abstract}
We study the temporal dynamics of photoexcited carriers in distributed Bragg reflector based semiconductor micropillars at room temperature. Their influence on the process of coherent phonon generation and detection is analyzed by means of pump-probe microscopy. The dependence of the measured mechanical signatures on laser-cavity detuning is explained through a model that accounts for the varying light-cavity coupling existent during the ultrashort times that pump and probe pulses dwell within the structure. To do so, we first explain the optical mode dynamics with an electron-hole diffusion model that accounts for the escape of carriers from the probed area, as well as their recombination in the bulk and on the free surfaces. We thus show that the latter is the most influential factor for pillars below $\sim 10 \mu \mathrm{m}$, where 3D confinement of the optical and mechanical fields becomes relevant.
\end{abstract}

DOI: 10.1103/PhysRevA.98.013816

\section{INTRODUCTION}

Motivated by the race for reaching the quantum regime in mesoscopic mechanical systems [1-5], research in cavity optomechanics has focused on the development of resonators with higher mechanical working frequencies [6-10]. Recent reports $[11,12]$ on resonators working beyond $10 \mathrm{GHz}$ reveal a new challenge in the field: the development of experimental techniques to study such high-frequency regimes. Suitable experimental techniques to study optomechanical resonators at mechanical frequencies of tens of GHz in the NIR-visible range are not yet accessible. Classical optomechanical experiments at low frequencies are based on Brownian motion measurements, which are done by impinging the sample with a continuous wave laser and measuring the RF noise spectral density of the transmitted/reflected light. These kinds of experiments give information about the displacement intensities. The method, provided the photodetector is fast and sensitive enough, could indeed allow the detection of vibrations up to tens of $\mathrm{GHz}$. State-of-the-art telecom wavelength photodetectors can be quite fast (up to $50 \mathrm{GHz}$ ) and by cooling them, it could be possible to lower their thermal noise, thus improving their sensitivity. NIR-visible light photodetectors, however, have not yet attained such a performance. Information about the vibrational dynamics can be obtained from ring-down techniques [13], recently applied in the domain of optomechanics [14-17]. In this case the use of ultrafast detectors is also necessary; hence the technique suffers the same bandwidth limitation as that of the Brownian motion measurements. Stroboscopic techniques appear as a potential solution, but their use remains limited in the context of optomechanics.

Usually used in nanophononics, pump-probe coherent phonon generation provides valuable information about the

\footnotetext{
*Corresponding author: afains@cab.cnea.gov.ar
}

eigenstates of the system, and their temporal evolution. This technique can provide full information about the coherent phonon dynamics up to $\mathrm{THz}$ frequencies [18-20]. However, in contrast to the other mentioned techniques, it requires a detailed knowledge of the system under study. Historically, this technique was used to qualitatively characterize phonon dynamics and for nondestructive testing [21]. Nevertheless, the complexity of the processes involved in the experiments (in both generation and detection of coherent phonons) prevents a straightforward characterization of the phonon dynamics [22-24]. To be able to extract all the information available from the experiments, it is essential to understand the optoelectronic system as well as the mechanisms involved in the measurements. In this paper we give the tools to decouple what are the effects of the carrier dynamics on the optical behavior of the system, from the mechanically induced modulation of the signals, showing the potential of the technique for optomechanics.

\section{RESULTS}

Since the interaction between electromagnetic fields in the NIR-visible range and mechanical vibrations are mediated, one way or the other, by electrons [19], understanding their behavior and what is exactly their influence on the measurements is imperative. In this work we focus on the photoexcited carrier dynamics and their relation with those of the mechanical and optical cavity modes. This is achieved by means of pumpprobe microscopy experiments, performed on square section micropillars with side lengths ranging from $2 \mu \mathrm{m}$ to $60 \mu \mathrm{m}$, and for several different laser detunings with respect to the fundamental optical cavity mode. The resonators studied here were dry-etched from a $\lambda / 2$ bulk-GaAs planar cavity enclosed between two distributed Bragg reflectors (DBRs) consisting of alternating $\mathrm{Ga}_{0.9} \mathrm{Al}_{0.1} \mathrm{As} / \mathrm{Ga}_{0.05} \mathrm{Al}_{0.95} \mathrm{As} \lambda / 4$ layers, 28 pairs at the bottom, and 24 on top. The resulting pillars can 


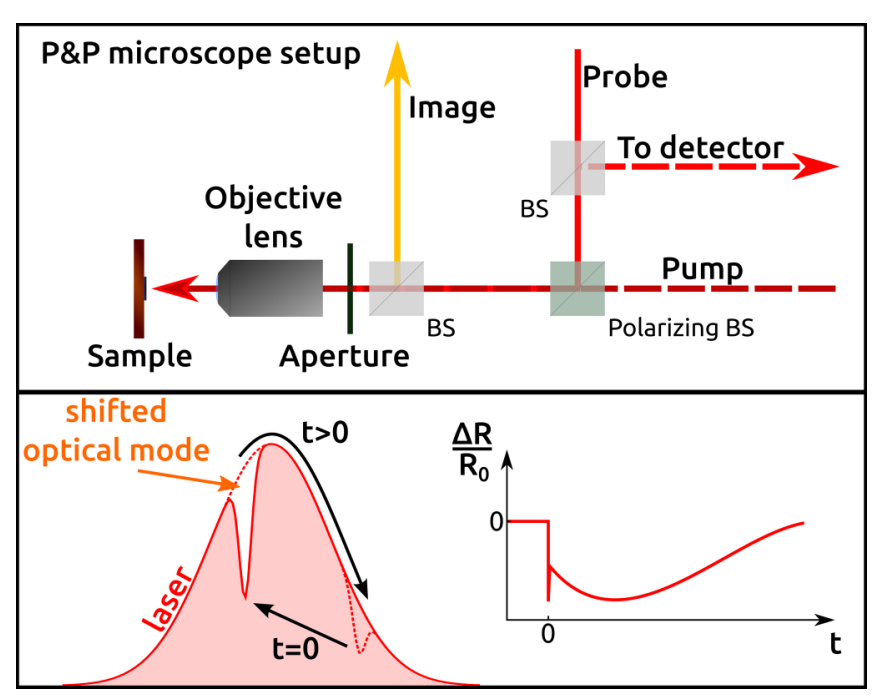

FIG. 1. Top: Pump-probe microscopy experimental setup. The polarizing beam splitter allows us to separate the reflections corresponding to pump and probe pulses, which are cross-polarized. The reflected probe pulse is guided either to a photodiode (to measure the differential reflectivity) or to a spectrometer (to measure the reflectivity spectrum). Bottom: Outline representing the effect the optical cavity mode has on the reflected probe spectra (left) and how the shifting mode changes the differential reflectivity measurement (right), which is based on the spectral integral of the reflected probe pulse. The reflectivity is minimum when the optical cavity mode is better coupled with the laser and the sample absorbs more. Any phonons present will also modulate the reflectivity [11].

confine the electromagnetic field and mechanical vibrations in the three dimensions [11], corresponding to the optical and acoustic cavity modes, respectively. To study the evolution of these systems after pulsed laser excitation, we implement a time-resolved differential optical reflectivity (pump-probe) measurement $[25,26]$ with micrometer resolution. In these experiments, two $\sim 2$ ps pulses ( $\sim 2 \mathrm{~nm}$ spectral width) of different intensity and crossed polarization are focused on the same spot. The pump ( $5 \mathrm{~mW}$ of incident power) is used to excite the sample, while the probe $(0.5 \mathrm{~mW}$ of incident power), delayed from the former, is used to measure the change generated in the optical reflectivity. The reflected probe pulse can be either guided to a photodiode or to a spectrometer. In the first case, the measurement will correspond to the total intensity change of the pulse (differential reflectivity), while in the latter a spectral analysis of the sample reflectivity can be obtained (see Appendix A). This is schematized in Fig. 1. Modifying the delay between the pump and probe pulses, it is possible to reconstruct the temporal evolution of the optical reflectivity.

When working with a laser wavelength near the GaAs electronic transition $(\sim 872 \mathrm{~nm})$, the pump pulse promotes electron-hole (e-h) pairs in the GaAs spacer in an area given by the excited spot. These carriers modify the refractive index of the material, changing the spectral position of the optical mode "seen" by the probe, which is sensing the same area. The reflected probe, being cross-polarized with respect to the pump by design, is separated from the latter using a polarizing beam splitter and is guided to a photodiode, to measure the differential reflectivity, or to a spectrometer, to measure the reflectivity spectrum within the spectral width of the probe pulse. In Fig. 2 we present the reflectivity of a $5 \mu \mathrm{m}$ square pillar [panel (a)], as well as the corresponding differential reflectivity [panel (b)], both as a function of delay time $(t)$. Here $t=0$ corresponds to the moment when the pump pulse hits the sample. Hence, at negative times the reflectivity corresponds to the unexcited state of the pillar, while at positive times we can see the temporal evolution of the reflectivity after excitation. The inset in Fig. 2(b) shows the laser spectrum, which is the same for the pump and the probe pulses. Four characteristic
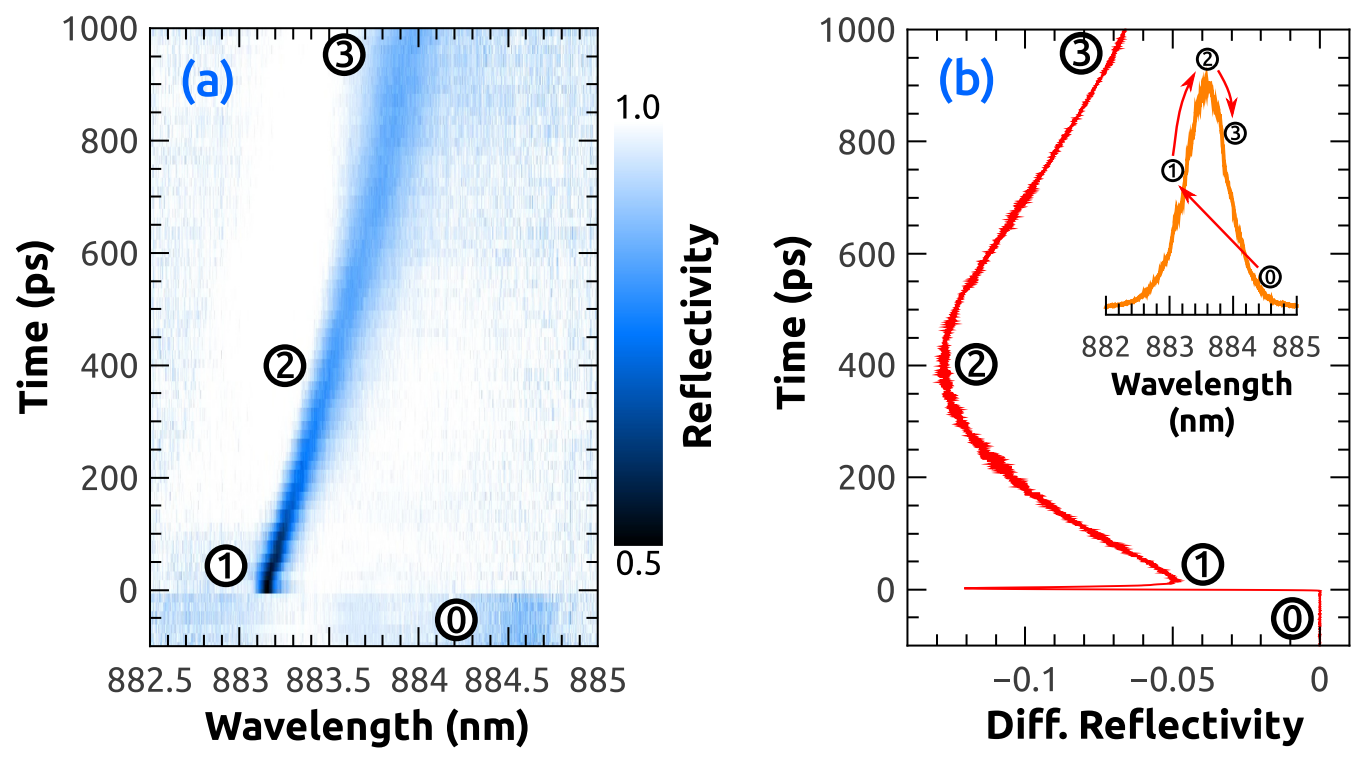

FIG. 2. (a) Optical mode spectral position and reflectivity as a function of time (map). The encircled numbers mark the spectral position of the optical mode for 4 characteristic times. (b) Differential reflectivity $\left(\Delta R / R_{0}\right)$ as a function of time. The black encircled numbers mark the same times and spectral positions as in (a). Inset: Pulsed laser spectrum (orange line). The red arrows are guides to the eye showing the spectral path followed by the optical mode between the characteristic times. 
times were marked with black encircled numbers to better understand the interrelation between the spectral dynamics and the differential reflectivity measurements. At $t<0$ the optical cavity mode at $\lambda_{c}=\lambda_{0} \sim 884.6 \mathrm{~nm}$ is red-detuned $\left(\lambda_{c}>\lambda_{\text {laser }}\right)$ with respect to the laser (label 0 in Fig. 2). When the pump pulse excites the sample the mode undergoes a strong shift, passing rapidly above the maximum of the laser spectral density and ending up blue-detuned (label 1) with respect to it. A deepening and narrowing of the optical mode resonance is observed right after pumping, which is due to a related decrease in the absorption of the spacer (see Appendix B). As the electronic system gradually recovers, the optical mode spectral position slowly returns to the maximum of the laser spectral density (label 2) and proceeds towards its initial state (label 3). These dynamics can be approximated with a steplike onset, followed by an exponential recovery:

$$
\begin{array}{ll}
\lambda_{c}(t)=\lambda_{0}, & t<0, \\
\lambda_{c}(t)=\lambda_{0}+\Delta \Lambda e^{-t / \tau_{e}}, & t \geqslant 0,
\end{array}
$$

$\lambda_{0}$ being the initial mode position, $\tau_{e}$ the characteristic recovery time, and $\Delta \Lambda$ the initial shift experienced by the mode.

In addition to the change in the optical properties, the photoexcited carriers generate coherent vibrations [19], corresponding to the mechanical eigenmodes of the structure [11]. These vibrations also perturb the optical mode, though to a much lesser degree than the carriers. Even though their effect is not observable in a spectroscopic measurement like the one presented in Fig. 2(a), they are readily detected when processing the differential reflectivity signal as shown in Fig. 2(b).

In Fig. 3 we present a set of differential reflectivity pumpprobe measurements performed on square section pillars with side lengths ranging from $2 \mu \mathrm{m}$ to $60 \mu \mathrm{m}$. Several curves per pillar are shown (same color), corresponding to different laser wavelengths, i.e., different detuning with respect to the optical cavity mode. A strong decrease of $\tau_{e}$ (indicated with the gray dashed curve) with decreasing pillar size is observed. Even though the traces are strongly dominated by the electronic contribution, hidden in them there are faint oscillations induced by the confined acoustic vibrations. These vibrations are generated by the photoexcited carriers through deformation potential interaction $[27,28]$, and can be singled out through Fourier analysis. To further understand the effect of lateral confinement on these vibrations, we selected, for each pillar, the trace corresponding to the specific detuning that leads to the absolute maximum in the amplitude of the measured oscillations. To these traces we then apply a rectangular bandpass spectral filter in order to select the fundamental mode alone $(\sim 19 \mathrm{GHz})$. Through this process, we get the traces shown in Fig. 4. The amplitudes as well as the lifetime of the mechanical modes show a significant dependence on size, something that will be the subject of a following article. Here we focus on the electronic response and the detuning dependence of the coherent generation and detection process. The traces show a $\pi$ phase shift [29] and a necking at a given delay $t_{\pi}$ (black circles in Fig. 4), which coincides with the passage of the optical mode over the maximum of the laser spectral density (labeled as 2 in Fig. 2), where the modulation on the reflected intensity due to the vibrations is negligible [29]. The exact delay at which it occurs depends both on the initial

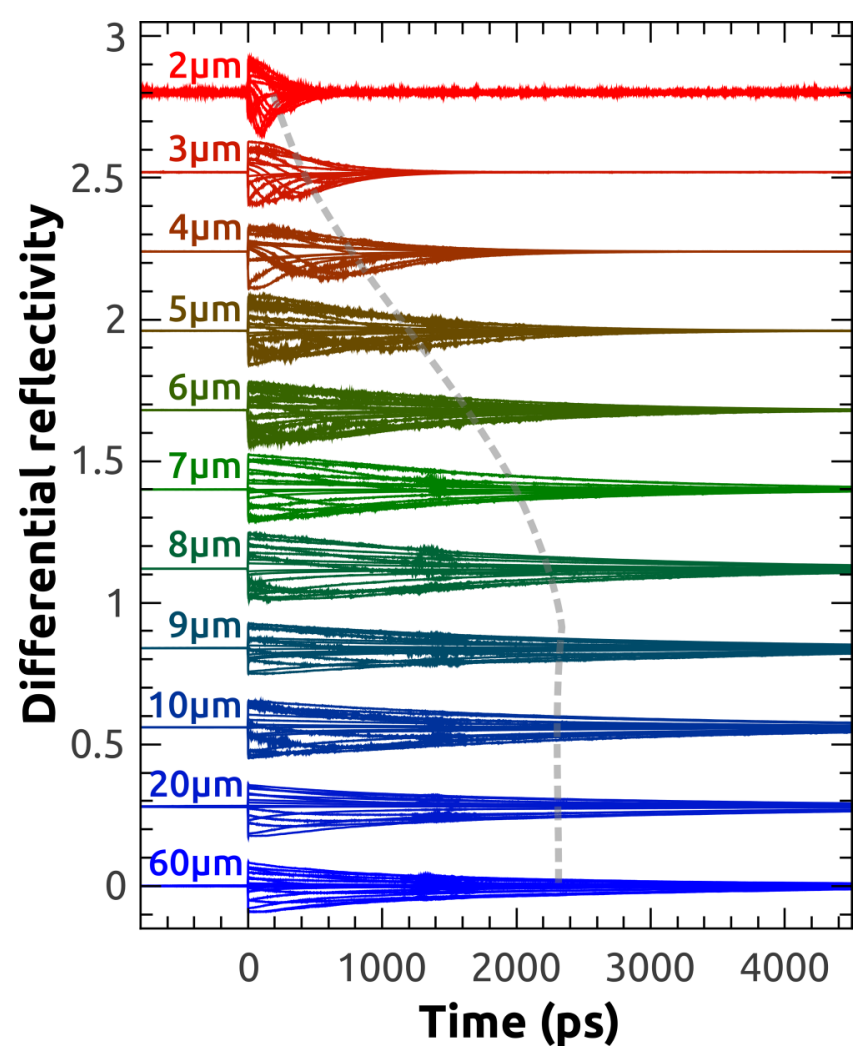

FIG. 3. Differential reflectivity as a function of time for pillars of different side lengths. The curves with the same color correspond to the same pillar at different detunings. The curves corresponding to different pillars are vertically shifted with respect to the rest for clarity. The gray dashed line marks the characteristic recovery time $\tau_{e}$.

detuning and on the recovery time $\tau_{e}$, and since $\tau_{e}$ depends on size, so does $t_{\pi}$. This dependence is especially clear for the smaller pillars in Fig. 4. The correlation is not so clear for the bigger pillars. In fact, $t_{\pi}$ for the two bigger pillars should appear at a time longer than the measuring window, for the detuning used in these experiments.

As is clear from Fig. 3 and Fig. 4, the optical mode dynamics strongly affect the sensitivity of the pump-probe technique to the lattice vibrations generated. This is evidenced by the fact that the vibrational modes' temporal dependencies, which should approximately follow an exponential decay, show a sort of beating instead, with a phase shift at $t_{\pi}$. This is only observed while the optical mode is spectrally shifting; once the mode is stable, the exponential decay behavior is evidenced, as is clearer for the smaller pillars (a more detailed explanation can be found in the Supplemental Material of Ref. [11]). We will focus on the carriers excited by the pump pulse, which are the responsible of the observed optical response. Once the carriers are excited, they diffuse in every direction within the GaAs spacer (the Al-rich slabs act as barriers) until they recombine. The recombination can happen in the bulk or the interfaces (with a given characteristic time $\tau_{e, 0}$ that we assume accounts for both) or on the free lateral surfaces of the pillars $\left(\tau_{e, 1}\right)$. Since on the latter the defect concentration is considerably higher than in the bulk or in the interfaces (which have molecular beam epitaxy quality), we assume $\tau_{e, 1} \ll \tau_{e, 0}$ [30]. Additionally to 


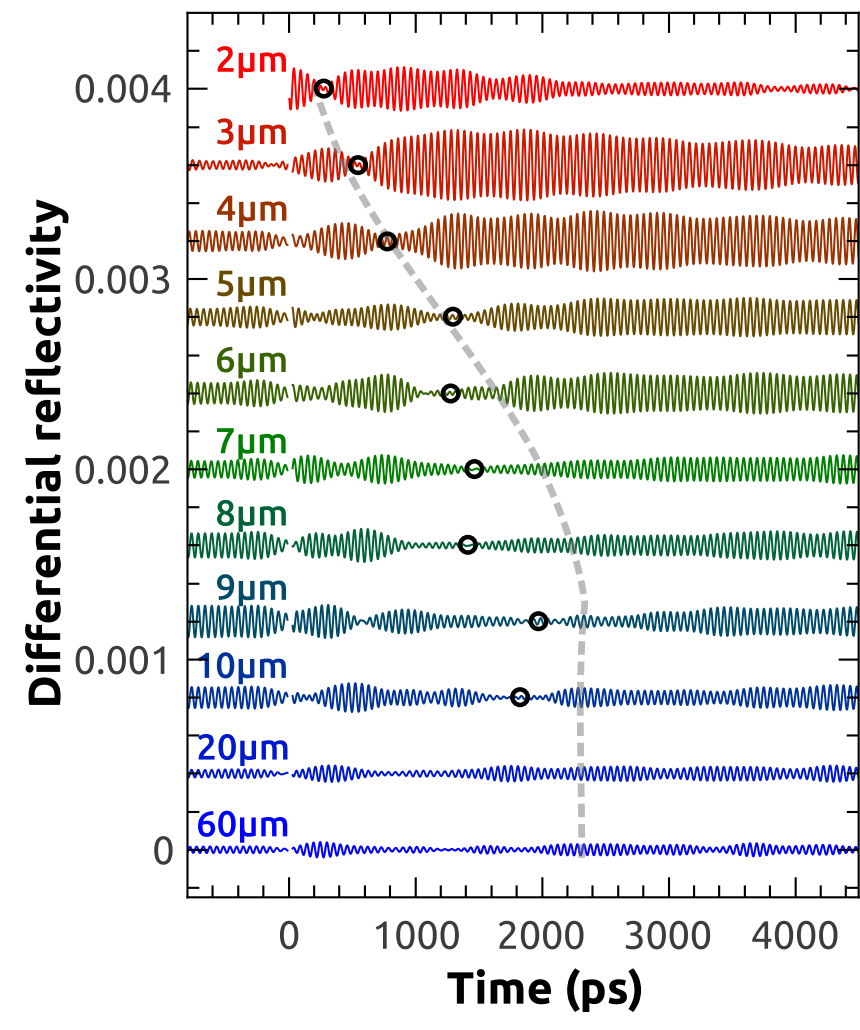

FIG. 4. Differential reflectivity oscillations due to the fundamental acoustical cavity mode $(\sim 19 \mathrm{GHz})$ for square section pillars of different sizes. Only the trace corresponding to optimum detuning is shown for each pillar. The curves corresponding to different pillars are vertically shifted with respect to the rest for clarity. The gray dashed line marks the characteristic recovery time $\tau_{e}$. The black circles mark the phase shift time $t_{\pi}$, corresponding to a $\pi$ phase change in the oscillations.

these recombination times, there is an effective decay time of the measured reflectivity signal $\left(\tau_{e, 2}\right)$, given by the carriers that diffuse out of the probed area. From measurements on a planar sample with a spot size of $\sim 50 \mu \mathrm{m}$, where there are no free surfaces and the latter diffusion effect is negligible, a value of $\tau_{e} \approx 7 \mathrm{~ns}$ was obtained. In addition, from measurements done in the same sample, but with a spot size of $\sim 4 \mu \mathrm{m}$, we found that $\tau_{e} \approx 2.2 \mathrm{~ns}$. This evidences the relevance of transverse diffusion of the photoexcited carriers $\left(\tau_{e, 2}\right)$ for the optical mode recovery, when a small spot size is used.

In Fig. 5 we show the experimental values (yellow circles) obtained for the characteristic recovery time $\tau_{e}$ as a function of pillar size (corresponding to the dashed curve in Fig. 3 and Fig. 4). An increase is observed as the side length grows, up to $L \sim 10 \mu \mathrm{m}$, above which no further variation is observed. To explain this behavior, we will propose a simple model which takes into account all the mechanisms described above (bulk and interface recombination, lateral diffusion, and free surface recombination). An initial Gaussian carrier distribution with a $4 \mu \mathrm{m}$ FWHM is considered, given by the shape of the pump laser spot. Regarding bulk and interface recombination, we use the experimental value obtained for the planar sample $\left(\tau_{e, 0}=7 \mathrm{~ns}\right)$. To fit the data, a diffusion constant $D=0.8 \frac{\mu \mathrm{m}^{2}}{\mathrm{~ns}}$ is assumed

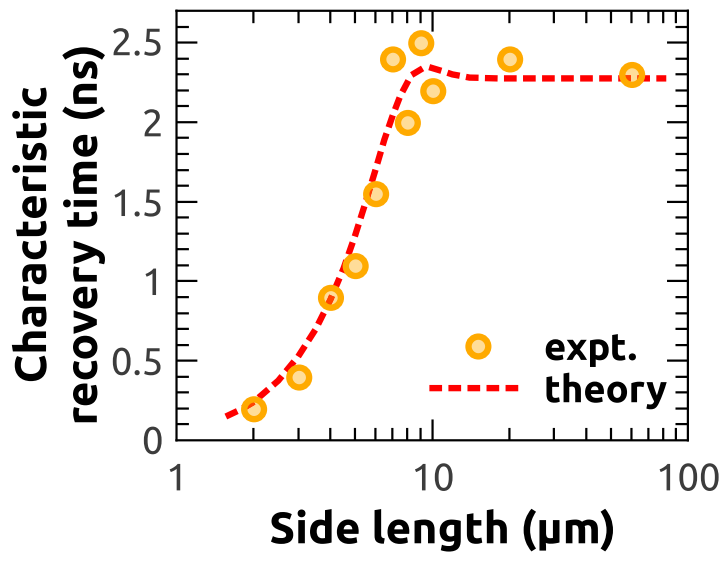

FIG. 5. Optical mode characteristic recovery time $\tau_{e}$ as a function of the side length $L$. Measurements (yellow circles) and theory (red dashed line).

[31]. The fast recombination on the free surfaces is simulated by forcing a zero concentration boundary condition, which corresponds to $\tau_{e, 1}=0$. In order to estimate the characteristic recovery time, we integrate the density of e-h pairs present in the area sensed by the probe pulse (same spot size as the pump pulse) as a function of time, and then fit the curve with an exponential decay, thus obtaining an estimate value for $\tau_{e}$ (see Appendix C). The theoretical result is displayed in Fig. 5 with the red dashed line. The agreement between data and theory is remarkable. The approximately constant value of $\tau_{e}$ observed for pillars with $L>10 \mu \mathrm{m}$ is determined, as in the case of the planar sample, by e-h diffusion out of the probed area. Below $L \approx 10 \mu \mathrm{m}$, the free surfaces start to play a more important role. As the side length decreases, the length the carriers have to travel in order to get there gets shorter. When the free surfaces are close enough for the e-h pairs to reach them in a time of the same order as the one they need to escape from the probed area ( $\tau_{e} \approx 2 \mathrm{~ns}$ ), their effect starts to be observable, and, hence, the effective decay time decreases. Note the detail in the transition zone, where the data show some spread and the simulated curve appears to have a maximum. This feature arises in a zone where the calculated curve for the carrier density as a function of time is not well fitted by a simple exponential decay. It happens because for those values of $L$ there is a change of regime, as we already mentioned, where the two characteristic times compete. Below and above that point the exponential decay approximation is quite accurate. The importance of the recovery time will be better understood later on. We will show how the ability to detect the vibrations present in the sample is closely related to it.

Regarding the mechanical vibrations, in Fig. 6 we present the intensity of the signature of the first acoustical cavity mode $(\sim 19 \mathrm{GHz})$ for four different pillar sizes as a function of laser wavelength (i.e., detuning). These intensities are obtained from the Fourier transform of the temporal traces. The intensities are normalized so that their maximum value is 1. Except for the blueshift of the curves due to the optical mode confinement in the smaller pillar sizes, there does not appear to be a significant difference in the behavior of the different pillars. Each curve presents two relative maxima separated by a dip, the one at higher energies being greater than the low-energy one. To 


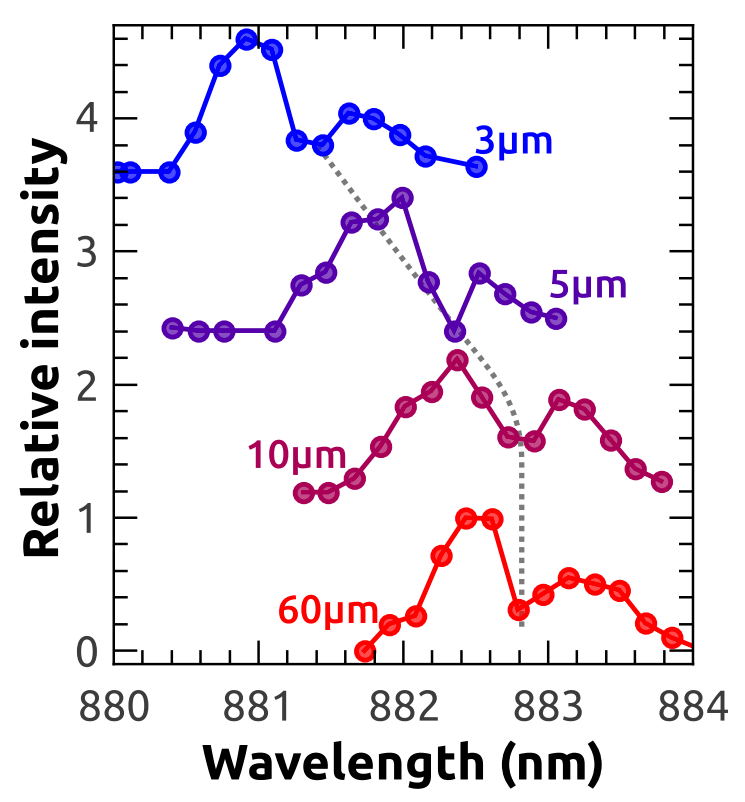

FIG. 6. Fundamental acoustic cavity mode dependence with laser wavelength for square pillars with side length of $3 \mu \mathrm{m}, 5 \mu \mathrm{m}, 10 \mu \mathrm{m}$, and $60 \mu \mathrm{m}$. The gray dotted line is a guide to the eye showing the approximate spectral position of the optical cavity mode. The curves were vertically shifted for clarity.

explain the observed behavior, we need to separate the origin of the measured coherent phonon intensity into two factors: generation efficiency and measurement sensitivity [22-24]. The former should be proportional to the photons absorbed by the sample from the pump pulse, while the latter is proportional to the change in the effective reflectivity due to any small change in the optical mode position (induced by the mechanical vibrations) with respect to the laser spectrum. Therefore, in a pulsed experiment, the generation would be expected to be more efficient at zero detuning, while the sensitivity should be maximum at zones of higher derivative, i.e., at the flanks of the laser spectral density. The two maxima seen in Fig. 6 are therefore related to the detection efficiency; the dip in between appears because of the null derivative at the laser maximum and is thus also a detection sensitivity feature.

The above conceptual arguments assume a frozen picture of the optical mode position. However, we have already shown that the cavity mode follows a complex dynamical behavior after pump excitation. Similar dynamics to those observed during recovery are expected to happen, though much faster and in the inverse direction, during excitation. In the following, we will address what is the consequence of these rapid dynamics on the efficiency of coherent phonon generation in pillar microcavities, as well as the role of the detection efficiency on the measured signals. To better understand this system, we will model the problem in a comprehensive way, taking into account the dynamics of the optical mode during the coherent phonon generation and detection, and the temporal dependence of the pump pulse inside the sample. We will assume that the number of phonons generated (Gen) is proportional to the total number of photoexcited carriers $N(\Delta t)$ ( $\Delta t$ being the total permanence time of the pump pulse in the cavity), which in turn is proportional to the total mode shift $\Delta \lambda_{c}(\Delta t)=\Delta \Lambda$
[30]:

$$
\text { Gen } \propto \Delta \Lambda \text {. }
$$

The total number of carriers excited after pumping, and therefore the total mode shift, depends on the initial mode position $\left(\lambda_{0}\right)$. Thus, the phonon generation will also depend on it:

$$
\operatorname{Gen}\left(\lambda_{0}\right) \propto \Delta \Lambda\left(\lambda_{0}\right) .
$$

To calculate this dependence, we consider a Gaussian pump pulse with a duration of $\sim 2$ ps and, using the transfer matrix method, we compute the electric field inside the GaAs spacer as a function of time $[E(t)]$. The electrons respond and are excited by the electric field with a delay time on the order of attoseconds [32]. Since we use picosecond pulses, we can assume their response to be instantaneous. The excited carriers change the refractive index of the sample, which in turn changes the spectral position $\left(\lambda_{i}\right)$ of the optical cavity modes [30]:

$$
\begin{aligned}
\Delta \lambda_{i}(t) & \propto \int\left|\mathcal{E}_{i}(\mathbf{r})\right|^{2} \mathcal{N}(\mathbf{r}, t) d \mathbf{r}=N(t) \int\left|\mathcal{E}_{i}(\mathbf{r})\right|^{2} n(\mathbf{r}) d \mathbf{r}, \\
\Delta \lambda_{i}(t) & \propto N(t) I_{i},
\end{aligned}
$$

where the subscript $i$ corresponds to the optical mode number, $\mathcal{E}_{i}(\mathbf{r})$ its corresponding spatial distribution, and $\mathcal{N}(\mathbf{r}, t)$ is the temporal and spatial dependence of the photocarrier density, which we assume separable into two functions, $n(\mathbf{r})$ and $N(t)$. $I_{i}$ thus defined is the overlap integral between the optical mode $i$ and the carrier distribution. The spectral change of the fundamental optical cavity mode at a given time $t$ will then be given by

$$
\frac{\partial \lambda_{c}(t)}{\partial t} \propto I_{c} \frac{\partial N(t)}{\partial t}=I_{c}|E(t)|^{2} \frac{N(t)-N_{\max }}{N_{\max }},
$$

$E(t)$ being the electric field of the incident pump pulse and $N_{\text {max }}$ the maximum photocarrier density, due to a possible saturation of the final states. Equation (4) expresses that the shift of the cavity mode at a given time $t$ is proportional to the density of photocarriers excited by the electric field. Applying this same concept to Eq. (5), it can rewritten as

$$
\frac{\partial \lambda_{c}(t)}{\partial t}=\alpha I_{c}|E(t)|^{2} \frac{\Delta \lambda_{c}(t)-\Delta \lambda_{\max }}{\Delta \lambda_{\max }},
$$

where

$$
\Delta \lambda_{c}(t)=\lambda_{c}(t)-\lambda_{0}
$$

Summing up, the variables that participate in the proposed model are the dependence on time of the electromagnetic field, given by the structure and the laser pulse (both known), the overlap integral $I_{c}$, the proportionality constant $\alpha$, and the maximum attainable mode shift $\left(\Delta \lambda_{\max }\right) . \Delta \lambda_{\max }$ corresponds to the maximum spectral shift which could be undergone by the optical mode, if $N_{\max }$ carriers were excited. This value is not necessarily reached experimentally, since the excitation depends both on the spectral distribution of the pump pulse and on the temporal dynamics of the optical mode. For example, if the excitation is initially very efficient, the optical mode will quickly pass over the region of high intensity of the laser (mode detuning $\approx 0$ ), and will end up in a region of low intensity (mode detuning $\ll 0$ ), where the excitation will decrease significantly. The strongest shift actually generated corresponds, in Fig. 7 , to a detuning of $\lambda_{0}-\lambda_{\text {laser }} \approx 0.85 \mathrm{~nm}$ 


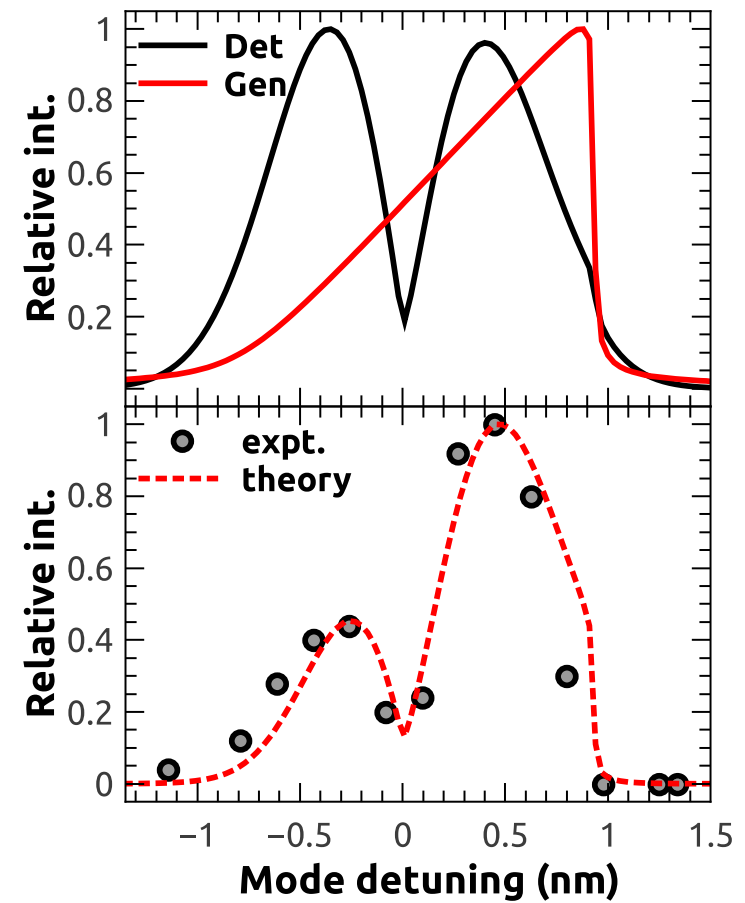

FIG. 7. Top: Generation (red) and detection (black) functions as calculated with the proposed model. Bottom: $\sim 19 \mathrm{GHz}$ acoustical cavity mode relative intensity as a function of optical mode detuning $\left(\lambda_{0}-\lambda_{\text {laser }}\right)$ for a $3 \mu \mathrm{m}$ square pillar (circles). The red dashed line corresponds to the theoretical calculation, given by the product between the generation (Gen) and detection (Det) functions.

(defined by the maximum of the red curve in the top panel). This value can be determined from spectral experiments such as the one shown in Fig. 2(a), which allows us to estimate the complete dependence for all detunings using the proposed approximations. Figure 7 shows the normalized (so that the maximum value is 1) generation function. A strong asymmetry is evident, which arises from the fact that the optical mode shifts to negative detunings. Therefore, if the initial detuning is positive the interaction between the cavity and the laser will be more efficient. On the other hand, if the initial detuning is negative, as soon as the mode shifts the laser cannot get into the cavity, and, therefore, the interaction will be weaker.

Having calculated $\Delta \Lambda\left(\lambda_{0}\right)$, which provides the detuning dependence of the amplitude of the generated vibrations, we need to find the dependence of the detection function $\operatorname{Det}\left(\lambda_{0}\right)$, given by the sensitivity of the probe pulse to these vibrations. This problem has already been considered by Sesin et al. [29] and Lanzillotti-Kimura et al. [22,23] in the low- $Q$ case (laser linewidth $<$ cavity mode width), for a planar structure. We apply the same concepts for our high- $Q$ (laser linewidth $>$ cavity mode width) micropillar, considering the other limit; i.e., taking the optical mode as infinitely narrow with respect to the laser linewidth. Furthermore, we take into account the fact that the optical cavity mode is not static, but is shifting due to the carrier population recovery after excitation. This means that the sensitivity is changing continuously over time, until the unexcited condition is recovered. Using the calculated optical shift and the experimental characteristic recovery time $\tau_{e}$ we calculate $\operatorname{Det}\left(\lambda_{0}\right)$. To do so, we average the detection sensitivity $\left(\left|\frac{\partial g(\lambda)}{\partial \lambda}\right|\right)$ during the measurement:

$$
\operatorname{Det}\left(\lambda_{0}\right) \propto \int_{0}^{t_{m}}\left|\frac{\partial g(\lambda)}{\partial \lambda}\right|_{\lambda_{c}(t)} d t,
$$

where $g(\lambda)$ is the spectral distribution of the laser intensity and $t_{m}$ is the experimental full-scan time. The resulting curve (also normalized so that its maximum value is 1) is presented in Fig. 7 (top) in black. It shows a more symmetric behavior as compared to the generation efficiency (red), with a minimum close to zero detuning due to the null derivative of the laser spectrum. A slightly higher relative maximum for negative detuning is observed. This can be understood from the fact that during recovery, if the initial detuning is positive, the mode will pass through the zero derivative zone, thus diminishing the average sensitivity.

Finally, we have an expression for both the generation and detection functions. Therefore, the intensity $\mathcal{I}\left(\lambda_{0}\right)$ of the measured vibrations can be obtained taking their product:

$$
\mathcal{I}\left(\lambda_{0}\right) \propto \operatorname{Gen}\left(\lambda_{0}\right) \operatorname{Det}\left(\lambda_{0}\right)
$$

In Fig. 7 we show $\operatorname{Gen}\left(\lambda_{0}\right)$, $\operatorname{Det}\left(\lambda_{0}\right)$, and $\mathcal{I}\left(\lambda_{0}\right)$, obtained for a $3 \mu \mathrm{m}$ square pillar, together with the experimental values. The generation and detection functions show, as was already remarked, very different behaviors. Their product, which gives the dependence of the intensity, shows an excellent agreement with the experimental data, as can be seen in Fig. 7 (bottom). Particularly, the different slopes at positive and negative detuning, as well as the relative intensity between the two maxima and the position of the central dip, are well reproduced. Moreover, this calculation enables us to understand what is the contribution to the measured signal coming from the generated vibrations, and what is due to the detection capability of the system.

\section{CONCLUDING REMARKS}

We have shown that the observed dynamics of the optical cavity mode are dominated by the diffusion of the photoexcited carriers out of the probed area for pillars bigger than $10 \mu \mathrm{m}$, and by surface recombination in the smaller ones. Using a diffusion model, we were able to reproduce this behavior with great accuracy. Moreover, we have been able to qualitatively explain the detuning dependence of the measured optomechanical response, separating the effects due to the dynamics of the optical mode on the phonon generation and on the detection efficiency. Our results explain satisfactorily well the experimental features observed. Particularly, the asymmetry in phonon intensity with detuning was shown to be due to the generation efficiency. In contrast with a static optical mode model during excitation, we show that the maximum in generation efficiency does not occur at zero detuning, but at positive detuning, because the mode shift follows the carrier excitation speed, thus being able to follow the fast electric field evolution inside the cavity due to the pump pulse. The detailed description of the generation and detection processes in DBR semiconductor micropillar cavities is an important step in the path to using these pump-probe techniques for the study of vibrational dynamics in a more quantitative way and in the study of complex phonon dynamics in semiconductor optomechanical systems at the micro- and nanoscales. 


\section{ACKNOWLEDGMENTS}

This work was partially supported by ANPCyT Grants PICT 2012-1661 and 2013-2047, the Labex NanoSaclay, the International Franco-Argentinean Laboratory LIFAN (CNRSCONICET), the French Agence Nationale pour la Recherche (grant ANR QDOM), the French RENATECH network, and ERC Starting Grant No. 715939 NanoPhennec.

\section{APPENDIX A: TIME-RESOLVED REFLECTION SPECTROSCOPY}

This technique is based on the spectral change that the probe pulses undergo when interacting with the sample. To extract useful information, the measurements must be properly processed. To this end, it is critical to understand the origin of the measured spectra and have a good representation of the unperturbed probe pulse.

The pump beam passes through an acousto-optic modulator, which lets through trains of $\sim 50$ pulses, with identical intervals without pulses. The probe beam is not modulated, and thus consists of a continuous train of pulses. For each time of the measurement, which lasts $\sim 1 \mathrm{~s}$ millions of probe pulses are collected and integrated, some that interacted with the excited sample, and others that interacted with the unexcited sample. That is, the measured spectrum will have both contributions simultaneously for all times. This is schematized in Fig. 8, where for simplicity, each train of pulses is represented with a single pulse. It shows how the spectra of the reflected probe

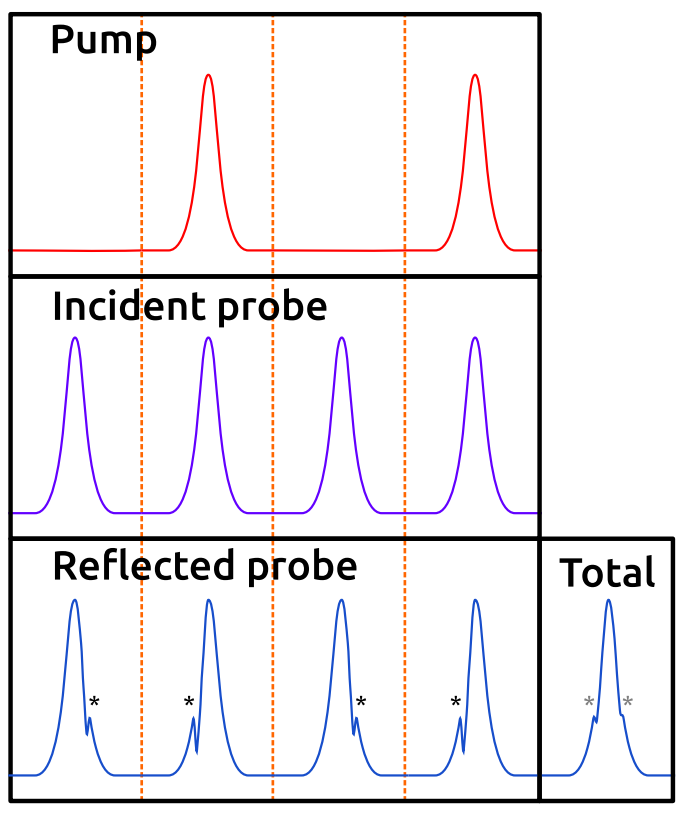

FIG. 8. Outline of the fundamentals of the experiment. The pump pulses (top) are blocked intermittently, while the probe pulses (center) are not. Because of this, the optical cavity mode, marked with an asterisk, is in different positions, and the probe pulses reflected from the sample (bottom) present a different spectrum, with a periodicity given by the modulation of the pump pulses. Since during the experiment we integrate during a timescale much longer than the period of the modulation, the measured spectra (bottom, right) are given by the average between the excited and unexcited reflectivity. pulses undergo a change given by the instantaneous position of the optical cavity mode (marked with an asterisk). The bottom rightmost box represents what is measured, due to the integration of the successive pulses. Let $\iota_{i}(\lambda)$ be the incident probe spectrum, $\iota_{0}(\lambda)=R_{0} \iota_{i}(\lambda)$ the reflected probe spectrum when the sample is not excited (first and third pulse in Fig. 8), and $\iota(\lambda, t)=R(t) \iota_{i}(\lambda)$ the reflected probe spectrum when the sample is being excited by the pump pulse (second and fourth pulse in Fig. 8).

The measured spectrum will, therefore, take the form

$$
\iota_{m}(\lambda, t)=\frac{P_{0} \iota_{0}(\lambda)+P \iota(\lambda, t)}{P_{0}+P},
$$

$P\left(P_{0}\right)$ being the number of pulses integrated with (without) excitation. Since the trains of pulses with and without excitation are integrated in series, the maximum possible difference between $P_{0}$ and $P$ is $\sim 100$. The total number of pulse trains integrated, on the other hand, is several million. Therefore, it is sensible to approximate $P_{0}=P$ :

$$
\iota_{m}(\lambda, t)=\frac{\iota_{0}(\lambda)}{2}+\frac{\iota(\lambda, t)}{2} .
$$

The measurement is done as a function of time, displacing the delay line. Therefore, there are probed times for which $\iota(\lambda, t) \approx$ $\iota_{0}(\lambda)$. This occurs when the time delay of the probe pulse is maximum $(\sim 12.5 \mathrm{~ns})$, which experimentally corresponds to the moment just before the pump-probe coincidence $\left(t=0^{-}\right)$. At that moment the measurement will have the form

$$
\iota_{m}\left(\lambda, 0^{-}\right) \approx \frac{\iota_{0}(\lambda)}{2}+\frac{\iota_{0}(\lambda)}{2}=\iota_{0}(\lambda)
$$

Therefore, using the $t=0^{-}$data it is possible to correct the measurement for every other time. The corrected measurement $\iota_{c}(\lambda, t)$ takes the form

$$
\begin{aligned}
& \iota_{c}(\lambda, t)=2\left(\iota_{m}(\lambda, t)-\frac{\iota\left(\lambda, 0^{-}\right)}{2}\right), \\
& \iota_{c}(\lambda, t) \approx 2\left(\frac{\iota_{0}(\lambda)}{2}+\frac{\iota(\lambda, t)}{2}-\frac{\iota_{0}(\lambda)}{2}\right), \\
& \iota_{c}(\lambda, t) \approx \iota(\lambda, t) .
\end{aligned}
$$

In this way, the contribution sought can be isolated and, finally, by dividing this corrected signal by the incident spectrum, the reflectivity of the sample at that time delay is obtained:

$$
R(\lambda, t)=\frac{\iota_{c}(\lambda, t)}{\iota_{i}(\lambda)} .
$$

To determine the reference incident pulse spectrum $\left[\iota_{i}(\lambda)\right]$, while maintaining the alignment and focus as stably as possible during the measurement, the following procedure was implemented. An algorithm was used that compares the spectrum before the coincidence $\left(t=0^{-}\right)$with the spectrum after the coincidence $\left(t=0^{+}\right)$. In this way, sections of the spectrum are selected where there are no disturbances due to the optical mode and the original spectrum is then reconstructed. The requirement that must be met so that this algorithm is accurate is that the spectral shift of the optical cavity mode must be greater than its linewidth. This is so, because if the comparison does not reveal the existing differences, it is not possible to correctly extract the incident spectrum, and therefore neither 

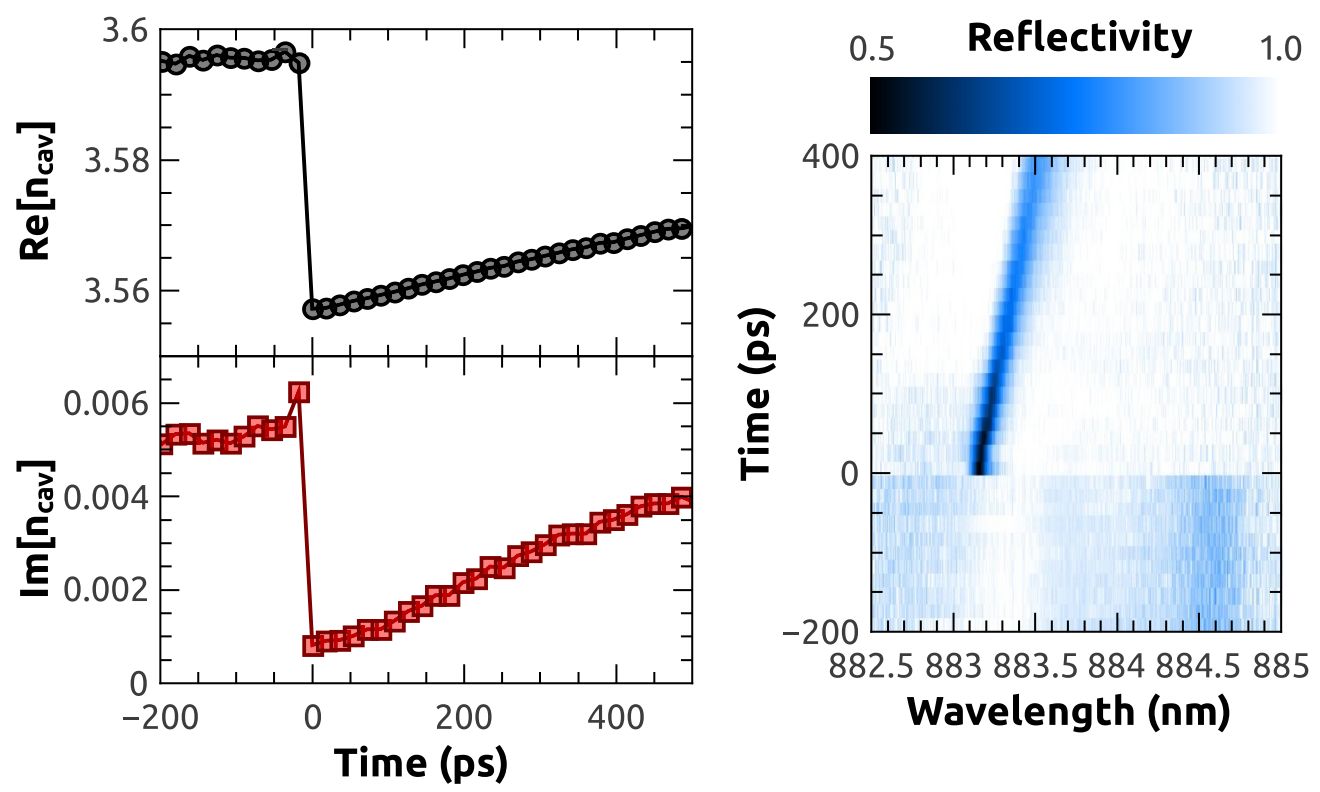

FIG. 9. The right panel shows the reflectivity spectrum as a function of time during the $200 \mathrm{ps}$ previous to the pump excitation, and the $400 \mathrm{ps}$ posterior to it. The left panel displays the temporal dependence of the real (top) and imaginary (bottom) components of the refractive index, as extracted from the reflectivity spectra.

the reflectivity. In our case, this means that the technique is limited to the study of pillars of side length smaller than $10 \mu \mathrm{m}$ and to spectral positions where the sample shows little absorption.

The major challenge of this method, beyond the difficulty in obtaining the incident spectrum, is that variations in the intensity of the probe, due to laser instabilities or to changes in the incidence position on the sample or in focus (mostly due to a small movement of the sample or to the unavoidable movement of the delay line), will make the normalization less effective for times far away from the coincidence.

\section{APPENDIX B: DETERMINATION OF THE REFRACTIVE INDEX TEMPORAL DEPENDENCE}

From the time-resolved reflection spectroscopy, it is possible to extract valuable information. For example, by fitting the experimental results with a matrix-method-based reflectivity model, the complex refractive index temporal dependence can be obtained. Typical results are presented in Fig. 9, along with the reflectivity measurements used for the fit. Because of the temporal constraints mentioned in the previous section, only $200 \mathrm{ps}$ before and $400 \mathrm{ps}$ after the coincidence were used for the fit. A time previous to the coincidence is chosen to set the fixed parameters of the model. Namely, the refractive index of GaAs at room temperature is taken from the literature [33] and the spacer and DBR layer thicknesses are proportionally adjusted to fit the position of the calculated mode to the experimental one. The imaginary component of the refractive index is then adjusted to fit the width of the mode. This done, the variable parameters used are the changes in the real and imaginary parts of the refractive index as a function of time delay. These are fitted by the least squares method for every time considered. The results show a decrease in both the real and imaginary components of the refractive index as the pump hits the sample, with a subsequent recovery to the unexcited condition. The change in the real part governs the negative shift in the spectral position of the mode, while the change in the imaginary part (directly related to the absorption) dominates the narrowing of the optical mode just after excitation (right panel in Fig. 9).

\section{APPENDIX C: CHARACTERISTIC RECOVERY TIME AND CARRIER DIFFUSION MODEL}

As shown in Appendix B, the refractive index of the GaAs spacer is subject to a strong change during pump excitation, followed by a relatively slow recovery. In this section we will consider the processes that govern this recovery. In particular, we will focus on the real component of the refractive index, as it is the one that has the stronger impact on the differential reflectivity measurements.

The signals measured by ultrafast reflectometry depend strongly on the size of the pillars; in the following, we will delve into this topic. First, we will address the dependence of the characteristic recovery time of the electronic system. The time it takes a pillar of a certain side length to recover its initial optical properties increases for bigger pillars. To parametrize this "recovery time," numerical fits were performed on the differential reflectivity measurements, assuming that the laser has a Gaussian spectral distribution and, as shown in Fig. 9, that the refractive index of the spacer changes sharply when the pump pulse excites the sample (the DBRs are left unchanged), and then recovers its original value with an exponential decay function with a characteristic recovery time $\tau_{e}$ :

$$
n(t)=n_{0}-\Delta n \Theta(t) e^{-\frac{t}{\tau_{e}}},
$$

$\Theta(t)$ being the Heaviside step function. To perform the fit, the reflectivity of the sample as a function of time is calculated by the transfer matrix method, and the result is convoluted with a Gaussian (obtained by fitting the laser spectrum) to obtain the 


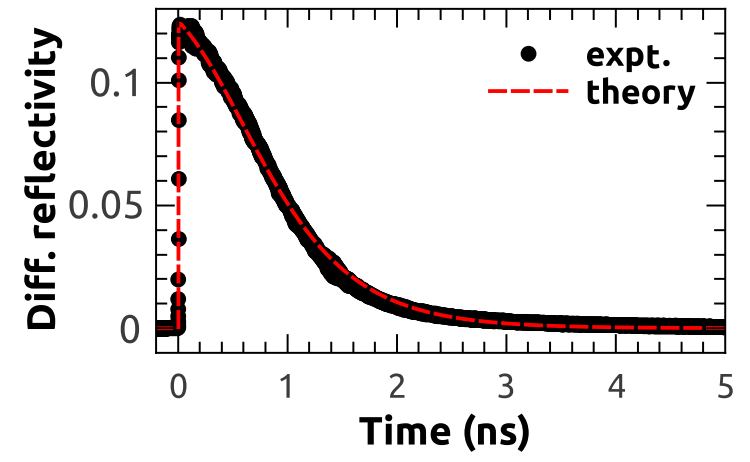

FIG. 10. $5 \mu \mathrm{m}$ square section pillar differential reflectivity measurement (black dots) and fit (red dashed line).

differential reflectivity as a function of time. An example of the result for a $5 \mu \mathrm{m}$ square section pillar is presented in Fig. 10. In this way, we derive from the differential reflectivity traces the GaAs spacer refractive index temporal dependence, which follows the characteristic recovery times of the electronic system. The obtained values for $\tau_{e}$ are presented in Fig. 5 as a function of side length (circles).

In the following we will address the model behind the theoretical results shown in Fig. 5 (red dashed line). The simulation takes into account the diffusion and recombination of the photoexcited carriers. To address this model, we first comment on some experimental results obtained in a planar sample. Differential reflectivity measurements were performed on a planar sample with two different experimental setups. In one experiment, we used a spot with a diameter of $\sim 50 \mu \mathrm{m}$, while in the other the spot was only $\sim 4 \mu \mathrm{m}$ in diameter (obtained by focusing the laser with a microscope objective). The characteristic recovery time of the electronic system was observed to depend on the spot diameter. Namely, $\tau_{e} \approx 7 \mathrm{~ns}$ for the $50 \mu \mathrm{m}$ spot, while $\tau_{e} \approx 2.3 \mathrm{~ns}$ for the $4 \mu \mathrm{m}$ one. Because we measure the specular reflection of the probe beam, the only relevant changes are those that occur within the probed area. Since the observed changes in the refractive index occur due to the presence of excited carriers in the spacer, if they diffuse laterally from the measured area, as far as the reflectivity is concerned, it will be equivalent to them having recombined. This is observed as a faster recovery of the refractive index. If the laser spot is sufficiently large, the effects of diffusion will be negligible (since the circumference to area ratio is smaller), and the changes observed will be due mainly to the recombination of the carriers.

Having established that the lateral carrier diffusion is relevant for the measurements obtained with the microscopy setup, we address next the situation evidenced in the micropillar samples. In this case, in addition to the lateral carrier diffusion, there also exists a physical limit, given by the edges. In Fig. 11 a scheme representing the initial distribution of photoexcited carriers and their rapid diffusion is presented. A key factor to consider is the lateral surfaces of the pillars. Due to the manufacturing process, they have a higher concentration of defects, which act as preferential centers for scattering and nonradiative recombination. The half-life of an electron-hole pair in the presence of these defects is on the order of a few ps [30]. Therefore, since our measurements last several ns,

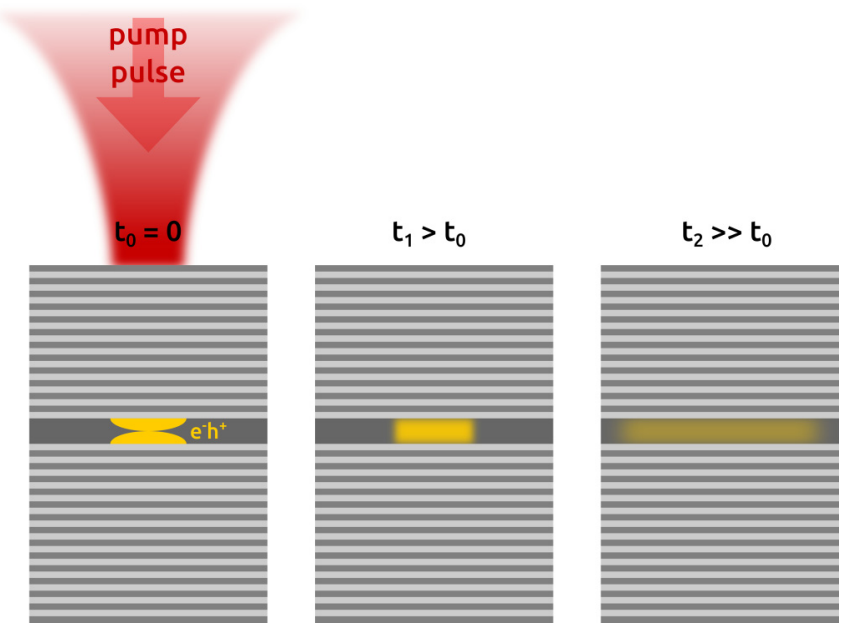

FIG. 11. Scheme of the distribution of the electron-hole pairs excited by the pump pulse (left), after diffusing for a short time (center), and a long time (right). The drawing is not to scale.

we will approximate this half-life to 0 . This is equivalent to considering a zero carrier concentration boundary condition at the lateral surfaces.

For the calculations we adopt the following hypothesis: (a) the carriers are initially distributed laterally with a Gaussian function given by the area excited by the pump pulse (coordinates referenced to the center of the pillar):

$$
N(x, y, t=0)=N_{0} e^{-\frac{\left(x^{2}+y^{2}\right)}{2 \sigma_{0}^{2}}},
$$

$\sigma_{0}$ being the initial standard deviation of the distribution; (b) the carrier concentration on the lateral surfaces is kept constant and equal to 0 :

$$
N\left( \pm \frac{L}{2}, y, t\right)=N\left(x, \pm \frac{L}{2}, t\right)=0
$$

(c) the change in the spectral position of the optical cavity mode is proportional to the number of carriers in the probed area A:

$$
\Delta \lambda(t) \propto \iint_{A} N(x, y, t) d x d y .
$$

Starting from these hypotheses, we must solve the diffusion equation:

$$
\frac{\partial N(x, y, t)}{\partial t}=D\left(\frac{\partial^{2} N(x, y, t)}{\partial x^{2}}+\frac{\partial^{2} N(x, y, t)}{\partial y^{2}}\right),
$$

where $D$ is the diffusion constant of electron-hole pairs in GaAs. The solution to this equation that complies with the initial conditions proposed has the form [34]

$$
\begin{aligned}
N(x, y, t) & =\frac{\sigma_{0}^{2} N_{0}}{\sigma^{2}(t)} e^{-\frac{\left(x^{2}+y^{2}\right)}{2 \sigma^{2}(t)},} \\
\sigma^{2}(t) & =\sigma_{0}^{2}+2 D t,
\end{aligned}
$$

where $\sigma(t)$ is the temporal dependence of the standard deviation of the carrier distribution. To obtain an analytical solution to this equation taking into account the boundary conditions we use virtual images outside the sample [34]. The solution 


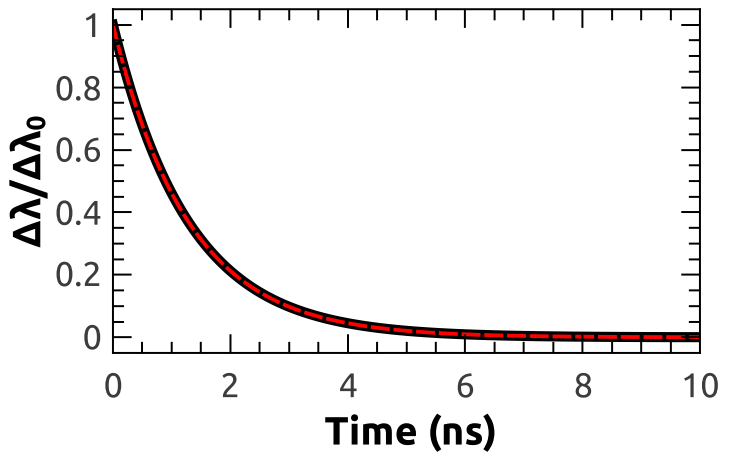

FIG. 12. Calculation of the temporal dependence of the optical cavity mode (normalized by the maximum shift) using Eq. (C9) (black thick line) and an exponential decay fit with a characteristic recovery time $\tau_{e} \approx 1.3 \mathrm{~ns}$ (red dotted line).

takes the form

$$
\begin{aligned}
N(x, y, t)= & \frac{\sigma_{0}^{2} N_{0}}{\sigma^{2}(t)}\left[\sum _ { n , m = - \infty } ^ { \infty } \left(e^{-\frac{(x+2 n L)^{2}+y^{2}}{2 \sigma^{2}(t)}}+e^{-\frac{x^{2}+(y+2 m L)^{2}}{2 \sigma^{2}(t)}}\right.\right. \\
& \left.\left.-e^{-\frac{[x+(2 n-1) L]^{2}+y^{2}}{2 \sigma^{2}(t)}}-e^{-\frac{x^{2}+[y+(2 m-1) L]^{2}}{2 \sigma^{2}(t)}}\right)-e^{-\frac{x^{2}+y^{2}}{2 \sigma^{2}(t)}}\right],
\end{aligned}
$$

where the first two terms of the summation are positive images and the next two terms are negative images. For mathematical convenience the real distribution is included inside this summation as a positive image. Since it is considered twice $(n=0$ and $m=0$ ), the last negative term is added to correct this. The need for multiple virtual sources of different sign arises from the fact that there are two edges per axis, so the images of one disturb the boundary condition of the other and vice versa, requiring corrections of higher order.

In addition to the diffusion we must take into account the recombination within the material. We will represent it by means of an experimentally obtained half-life, $\tau_{e, 0}=7 \mathrm{~ns}$. The distribution of carriers will then have the final form:

$$
\begin{aligned}
N(x, y, t)= & \frac{\sigma_{0}^{2} N_{0}}{\sigma^{2}(t)}\left[\sum _ { n , m = - \infty } ^ { \infty } \left(e^{-\frac{(x+2 n L)^{2}+y^{2}}{2 \sigma^{2}(t)}}+e^{-\frac{x^{2}+(y+2 m L)^{2}}{2 \sigma^{2}(t)}}\right.\right. \\
& \left.\left.-e^{-\frac{[x+(2 n-1) L]^{2}+y^{2}}{2 \sigma^{2}(t)}}-e^{-\frac{x^{2}+[y+(2 m-1) L]^{2}}{2 \sigma^{2}(t)}}\right)-e^{-\frac{x^{2}+y^{2}}{2 \sigma^{2}(t)}}\right] e^{-\frac{t}{\tau_{e, 0}}}
\end{aligned}
$$

In order to obtain the temporal dependence of the spectral position of the optical cavity mode, it is then necessary to replace Eq. (C8) in Eq. (C4) and integrate, obtaining

$$
\begin{aligned}
\Delta \lambda(t) \propto & \left\{\Gamma\left(\frac{R}{\sqrt{2} \sigma(t)}\right)+\frac{1}{2} \sum_{n=1}^{\infty}(-1)^{n}\left[\Gamma\left(\frac{n L+R}{\sqrt{2} \sigma(t)}\right)\right.\right. \\
& \left.\left.-\Gamma\left(-\frac{n L+R}{\sqrt{2} \sigma(t)}\right)+\Gamma\left(-\frac{n L-R}{\sqrt{2} \sigma(t)}\right)-\Gamma\left(\frac{n L-R}{\sqrt{2} \sigma(t)}\right)\right]\right\} \\
& \times e^{-\frac{t}{\tau_{0}}}
\end{aligned}
$$

Here $R$ is the radius of the probed area and $\Gamma$ the error function. Plotting Eq. (C9) as a function of time we obtain a curve like the one presented in black in Fig. 12. The red dotted line is an exponential decay fit with a characteristic recovery time $\tau_{e} \approx 1.3 \mathrm{~ns}$. The example presented in Fig. 12 corresponds to a $5 \mu \mathrm{m}$ pillar, corresponding to one point of the red dashed curve shown in Fig. 5. The parameters used to generate Fig. 12 were

$$
\begin{aligned}
L & =5 \mu \mathrm{m}, \quad R=1.7 \mu \mathrm{m}, \\
D & =0.8 \frac{\mu \mathrm{m}^{2}}{\mathrm{~ns}}, \quad \sigma_{0}=1.7 \mu \mathrm{m}, \\
\tau_{0} & =7 \mathrm{~ns}
\end{aligned}
$$

Applying Eq. (C9) for different values of $L$ (keeping all other parameters fixed) and repeating the procedure exemplified in Fig. 12, the red dashed line presented in Fig. 5 was obtained.
[1] S. Gröblacher, K. Hammerer, M. R. Vanner, and M. Aspelmeyer, Nature (London) 460, 724 (2009).

[2] J. D. Teufel, D. Li, M. S. Allman, K. Cicak, A. J. Sirois, J. D. Whittaker, and R. W. Simmonds, Nature (London) 471, 204 (2011).

[3] J. D. Thompson, B. M. Zwickl, A. M. Jayich, F. Marquardt, S. M. Girvin, and J. G. E. Harris, Nature (London) 452, 72 (2008).

[4] G. Anetsberger, O. Arcizet, Q. P. Unterreithmeier, R. Rivière, A. Schliesser, E. M. Weig, J. P. Kotthaus, and T. J. Kippenberg, Nat. Phys. 5, 909 (2009).

[5] A. D. O'Connell, M. Hofheinz, M. Ansmann, R. C. Bialczak, M. Lenander, E. Lucero, M. Neeley, D. Sank, H. Wang, M. Weides, J. Wenner, J. M. Martinis, and A. N. Cleland, Nature (London) 464, 697 (2010).

[6] L. Ding, C. Baker, P. Senellart, A. Lemaitre, S. Ducci, G. Leo, and I. Favero, Phys. Rev. Lett. 105, 263903 (2010).
[7] X. Sun, X. Zhang, and H. X. Tang, Appl. Phys. Lett. 100, 173116 (2012).

[8] R. Van Laer, B. Kuyken, D. Van Thourhout, and R. Baets, Nat. Photonics 9, 199 (2015).

[9] T. Carmon and K. J. Vahala, Phys. Rev. Lett. 98, 123901 (2007).

[10] M. Tomes and T. Carmon, Phys. Rev. Lett. 102, 113601 (2009).

[11] S. Anguiano, A. E. Bruchhausen, B. Jusserand, I. Favero, F. R. Lamberti, L. Lanco, I. Sagnes, A. Lemaître, N. D. LanzillottiKimura, P. Senellart, and A. Fainstein, Phys. Rev. Lett. 118, 263901 (2017).

[12] F. R. Lamberti, Q. Yao, L. Lanco, D. T. Nguyen, M. Esmann, A. Fainstein, P. Sesin, S. Anguiano, V. V. Villafañe, A. Bruchhausen, P. Senellart, I. Favero, and N. D. Lanzillotti-Kimura, Opt. Express 25, 24437 (2017).

[13] J. Güttinger, A. Noury, P. Weber, A. M. Eriksson, C. Lagoin, J. Moser, C. Eichler, A. Wallraff, A. Isacsson, and A. Bachtold, Nat. Nanotechnol. 12, 631 (2017). 
[14] N. Ares, T. Pei, A. Mavalankar, M. Mergenthaler, J. H. Warner, G. A. D. Briggs, and E. A. Laird, Phys. Rev. Lett. 117, 170801 (2016).

[15] P. Weber, J. Güttinger, A. Noury, J. Vergara-Cruz, and A. Bachtold, Nat. Commun. 7, 12496 (2016).

[16] D. Kleckner, B. Pepper, E. Jeffrey, P. Sonin, S. M. Thon, and D. Bouwmeester, Opt. Express 19, 19708 (2011).

[17] R. Dahan, L. L. Martin, and T. Carmon, Optica 3, 175 (2016).

[18] O. Matsuda, M. C. Larciprete, R. L. Voti, and O. B. Wright, Ultrasonics 56, 3 (2015).

[19] P. Ruello and V. E. Gusev, Ultrasonics 56, 21 (2015).

[20] A. Huynh, M. F. P. Winter, B. Perrin, B. Jusserand, A. Lemaître, and A. Fainstein, J. Acoust. Soc. Am. 123, 3549 (2008).

[21] M. C. Fischer, J. W. Wilson, F. E. Robles, and W. S. Warren, Rev. Sci. Instrum. 87, 31101 (2016).

[22] N. D. Lanzillotti-Kimura, A. Fainstein, B. Perrin, and B. Jusserand, Phys. Rev. B 84, 064307 (2011).

[23] N. D. Lanzillotti-Kimura, A. Fainstein, B. Perrin, B. Jusserand, L. Largeau, O. Mauguin, and A. Lemaitre, Phys. Rev. B 83, 201103 (2011).

[24] Y. Li, Q. Miao, A. V. Nurmikko, and H. J. Maris, J. Appl. Phys. 105, 083516 (2009).
[25] C. Thomsen, H. T. Grahn, H. J. Maris, and J. Tauc, Phys. Rev. B 34, 4129 (1986).

[26] A. Bartels, T. Dekorsy, H. Kurz, and K. Köhler, Phys. Rev. Lett. 82, 1044 (1999).

[27] S. A. Akhmanov and V. E. Gusev, Sov. Phys. Usp. 35, 153 (1992).

[28] V. Villafañe, P. Sesin, P. Soubelet, S. Anguiano, A. E. Bruchhausen, G. Rozas, C. G. Carbonell, A. Lemaître, and A. Fainstein, Phys. Rev. B 97, 195306 (2018).

[29] P. Sesin, P. Soubelet, V. Villafañe, A. E. Bruchhausen, B. Jusserand, A. Lemaître, N. D. Lanzillotti-Kimura, and A. Fainstein, Phys. Rev. B 92, 075307 (2015).

[30] H. Thyrrestrup Nielsen, E. Yuce, G. Ctistis, J. Claudon, W. Vos, and J.-M. Gerard, Appl. Phys. Lett. 105, 111115 (2014).

[31] B. A. Ruzicka, L. K. Werake, H. Samassekou, and H. Zhao, Appl. Phys. Lett. 97, 262119 (2010).

[32] M. T. Hassan, T. T. Luu, A. Moulet, O. Raskazovskaya, P. Zhokhov, M. Garg, N. Karpowicz, A. M. Zheltikov, V. Pervak, F. Krausz, and E. Goulielmakis, Nature (London) 530, 66 (2016).

[33] J. S. Blakemore, J. Appl. Phys. 53, R123 (1982).

[34] J. Crank, in The Mathematics of Diffusion (Clarendon Press, Oxford, 1975). 